\title{
Review: mydriatic retinal photography is the most effective test for detecting diabetic retinopathy
}

\author{
Hutchinson A, McIntosh A, Peters J, et al. Effectiveness of screening and monitoring tests for diabetic retinopathy-a \\ systematic review. Diabet Med 2000 Jul;17:495-506.
}

\section{QUESTION: Which screening tests for diabetic retinopathy are most effective?}

\section{Data sources}

Studies (1983-99) were identified by searching CINAHL, the Cochrane Trials Register, EMBASE/ Excerpta Medica, HealthSTAR, Medline, PsycLIT, Science Citation Index, Social Science Citation Index, HEED, NHS Economic Evaluation Database for Economic Evaluations, ECRI HTAIS, HMIC, and SIGLE. Conference proceedings were identified by using the Index to Scientific and Technical Conference Proceedings.

\section{Study selection}

English language, published studies of type 1 and 2 diabetes mellitus were selected if they addressed screening for and early management of diabetic retinopathy. Inclusion criteria were a prospective study design, comparison of $\geqslant 1$ screening method in a blinded fashion with a reference standard, and all patients having had the reference examination.

\section{Data extraction}

Data were extracted on patients, health professionals, study design and setting, tests and reference standards, sensitivities and specificities, and type of retinopathy.

\section{Main results}

22 studies (no randomised controlled trials) met the inclusion criteria. Outcomes used were any type of retinopathy, background retinopathy, maculopathy, sight threatening retinopathy, referable retinopathy, proliferative retinopathy, and serious retinopathy. Various reference standards were used, such as ophthalmologists using ophthalmoscopy or retinal photography, or both; assessment by diabetes specialist physicians; and trained graders of retinal photographs. 2 screening tests, ophthalmoscopy (direct or indirect) and retinal photography (with or without mydriasis), were used. Ophthalmoscopy by health professionals to detect varying stages of retinopathy in patients with type 2 diabetes showed specificities $>91 \%$ with specificities being greater than the associated sensitivities. Sensitivities ranged between $25 \%-66 \%$ for general practitioners, $48 \%-82 \%$ for opticians or optometrists, $43 \%-79 \%$ for ophthalmologists, and $27 \%-73 \%$ for other health professionals. Use of retinal photographs by health professionals showed lower specificities and higher sensitivities than those achieved with ophthalmoscopy, with sensitivities being greater than the associated specificities. Sensitivities ranged between $87 \%-100 \%$ for general practitioners using mydriatic photographs, were $>91 \%$ for optometrists using mydriatic photographs, and ranged between 89\%-93\% for ophthalmologists or their assistants using mydriatic or non-mydriatic photographs.

\section{Conclusion}

Mydriatic retinal photography is the most effective test for detecting diabetic retinopathy, but when photographs are ungradable, the addition of indirect ophthalmoscopy is suggested.

\section{COMMENTARY}

Screening for diabetic retinopathy is supported by evidence that early interventions can prevent or delay vision loss in patients with diabetes. ${ }^{12}$ Screening tests should be highly sensitive for detecting most cases while minimising misclassification. Mydriatic retinal photography of appropriate quality is sensitive in most of the small and heterogeneous studies included in this well done systematic review. Alternatives include non-mydriatic retinal photography and ophthalmoscopy by an eye care health professional. The choice of screening method is more important than which appropriately trained health professional administers it as long as strict quality standards are used. The ideal screening interval appears to be between annually and triennially, depending on the patient's risk. ${ }^{3}$

Despite the evidence, up to $50 \%$ of patients with diabetes are not offered periodic retinopathy screening. ${ }^{4}$ Health professionals should make use of the increasing number of organised healthcare services for people with diabetes (eg, outreach mobile screening) ${ }^{5}$ and avoid missed opportunities to screen for diabetic retinopathy (eg, with several patient reminders) ${ }^{6}$ With the accumulating evidence about how to screen for diabetic retinopathy, every reason exists to implement this activity, thereby preventing blindness in some patients.

\section{Victor M Montori, MD Mayo Clinic Rochester Rochester, Minnesota, USA}

1 The Diabetic Retinopathy Study Research Group. Photocoagulation treatment of proliferative diabetic retinopathy. Clinical application of diabetic retinopathy study (DRS) findings, DRS report number 8. Ophthalmology 1981;88:583-600.

2 Early Treatment Diabetic Retinopathy Study Research Group. Photocoagulation for diabetic macular oedema. Early treatment diabetic retinopathy study report number 1. Arch Ophthalmol 1985;103:1796-806.

3 Vijan S, Hofer TP, Hayward RA. Cost-utility analysis of screening intervals for diabetic retinopathy in patients with type 2 diabetes mellitus. JAMA 2000;283:889-96.

4 Mayfield JA, Rith-Najarian SJ, Acton KJ, et al. Assessment of diabetes care by medical record review. The Indian Health Service model. Diabetes Care 1994:17:918-23.

5 James M, Turner DA, Broadbent DM, et al. Cost effectiveness analysis of screening for sight threatening diabetic eye disease. BMJ 2000;320:1627-31.

6 Halbert RJ, Leung KM, Nichol JM, et al. Effect of multiple patient reminders in improving diabetic retinopathy screening. A randomised trial. Diabetes Care 1999;22:752-5. 